\title{
Establishing the relationship between galaxies and dark matter
}

\author{
H. J. Mo ${ }^{1}$, X.H. Yang ${ }^{2}$, F.C. van den Bosch ${ }^{3}$, Y.P. Jing ${ }^{2}$, \\ and S.M. Weinmann ${ }^{4}$ \\ ${ }^{1}$ Department of Astronomy, University of Massachusetts, Amherst, MA 01003, USA \\ email: hjmo@nova.astro.umass.edu \\ ${ }^{2}$ Shanghai Astronomical Observatory, Shanghai, China \\ ${ }^{3}$ Max-Planck-Institute for Astronomy, Heidelberg, Germany \\ ${ }^{4}$ Institute of Astronomy, Zurich, Switzerland
}

\begin{abstract}
We use two methods to establish the relationship between galaxies and dark matter halos. One is based the conditional luminosity function model, which links galaxies and dark matter halos by matching the number density and clustering properties of galaxies with those of dark matter halos in the current CDM model. The second is based on galaxy systems identified from large redshift surveys of galaxies. The galaxy - dark halo relationships established by these two methods match well, and can provide important constraints on how galaxies form and evolve in the universe.
\end{abstract}

Keywords. Galaxies, dark matter, dark matter halos

\section{The Conditional Luminosity Function}

One powerful method to establish the galaxy/halo connection is to use the halo occupation distribution, $P(N \mid M)$, which gives the probability to find $N$ galaxies (with some specified properties) in a halo of mass $M$. Since the total luminosity (in a given band) is one of the most important physical properties of a galaxy, it is important to consider galaxy occupation statistics as a function of luminosity. In a series of papers (e.g. Yang et al. 2003a; van den Bosch et al. 2003a), we did this by introducing the conditional luminosity function $(\mathrm{CLF}), \Phi(L \mid M) \mathrm{d} L$, which gives the average number of galaxies with luminosities in the range $L \pm \mathrm{d} L / 2$ that reside in a halo of mass $M$. The CLF links the galaxy luminosity function $\Phi(L)$ and the halo mass function $n(M)$ through

$$
\Phi(L)=\int_{0}^{\infty} \Phi(L \mid M) n(M) \mathrm{d} M .
$$

In CDM cosmologies, more massive haloes are more strongly clustered (e.g., Mo \& White 1996). This means that information about the clustering strength of galaxies also contains information about the characteristic mass of the halo in which they reside. At sufficiently large separations, the two-point correlation function of galaxies of luminosity $L$ is given by $\xi_{\mathrm{gg}}(r, L)=\bar{b}^{2}(L) \xi_{\mathrm{dm}}(r)$. Here $\xi_{\mathrm{dm}}(r)$ is the dark matter mass correlation function, and $\bar{b}(L)$ is the average bias of galaxies of luminosity $L$, which can be derived from the CLF according to

$$
\bar{b}(L)=\frac{1}{\Phi(L)} \int_{0}^{\infty} \Phi(L \mid M) b(M) n(M) \mathrm{d} M,
$$

with $b(M)$ the bias of dark matter haloes of mass $M$. Therefore, the combination of an observed luminosity function, $\Phi(L)$, plus measurements of the galaxy-galaxy two-point correlation function as function of luminosity can put constraints on $\Phi(L \mid M)$. 

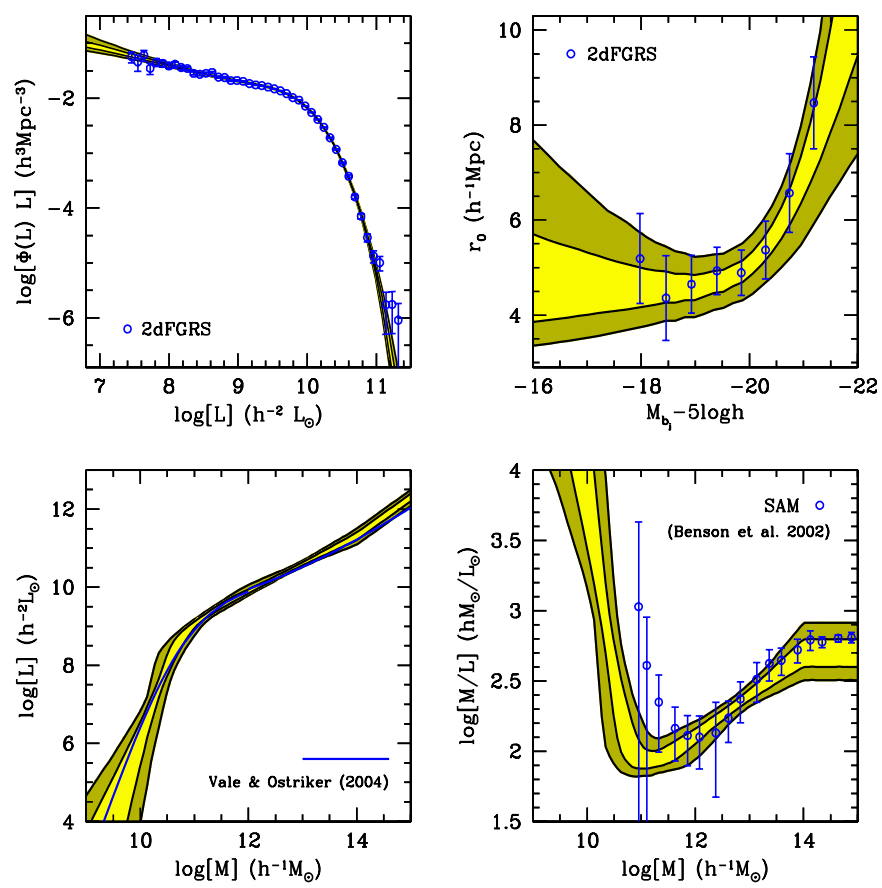

Figure 1. Posterior constraints on a number of quantities computed from the MCMC described in the text. The contours show the $68 \%$ and $99 \%$ confidence limits from the marginalized distribution. Upper left-hand panel: The galaxy luminosity function; open circles with errorbars correspond to the 2dFGRS data from Madgwick et al. (2002). Upper right-hand panel: galaxy-galaxy correlation lengths as function of absolute magnitude; open circles with errorbars correspond to the 2dFGRS data from Norberg et al. (2002). Lower left-hand panel: the total luminosity per halo as function of halo mass. Solid line corresponds to the model of (Vale \& Ostriker 2002), and is shown for comparison. Lower right-hand panel: the average mass-to-light ratio as function of halo mass. Open circles with errorbars correspond to the semi-analytical model of Benson et al. (2002) and is shown for comparison. See text for details.

We make the assumption that the CLF can be described by a Schechter function, and describe the mass dependency of the CLF using a total of 8 free parameters (e.g. van den Bosch et al. 2005). We use a Monte-Carlo Markov Chain (hereafter MCMC) to probe the likelihood function in our multi-dimensional parameter space, and to put confidence levels on all derived quantities. The results obtained for a $\Lambda \mathrm{CDM}$ 'concordance' cosmology $\left(\Omega_{m}=0.3, \Omega_{\Lambda}=0.7, h=0.7, \sigma_{8}=0.9\right)$ are shown in Fig. 1. The open circles with errorbars in the upper panels are the data used to constrain the models. The shaded areas indicate the 68 and 99 percent confidence levels on $\Phi(L)$ and $r_{0}(L)$ computed from the MCMC. Note the good agreement with the data, indicating that the CLF can accurately match the observed abundances and clustering properties of galaxies in the 2dFGRS. We emphasize that this is not a trivial result, as the data can only be fitted for a certain combination of cosmological parameters (see van den Bosch et al. 2003b).

The lower left-hand panel of Fig. 1 plots the relation between halo mass $M$ and the total halo luminosity $L$, which follows from the CLF according to

$$
\langle L\rangle(M)=\int_{0}^{\infty} \Phi(L \mid M) L \mathrm{~d} L
$$

Note that the confidence levels are extremely tight, especially for the more massive haloes. The $L(M)$ relation reveals a dramatic break at around $M \simeq 10^{11} h^{-1} \mathrm{M}_{\odot}$, indicating a 


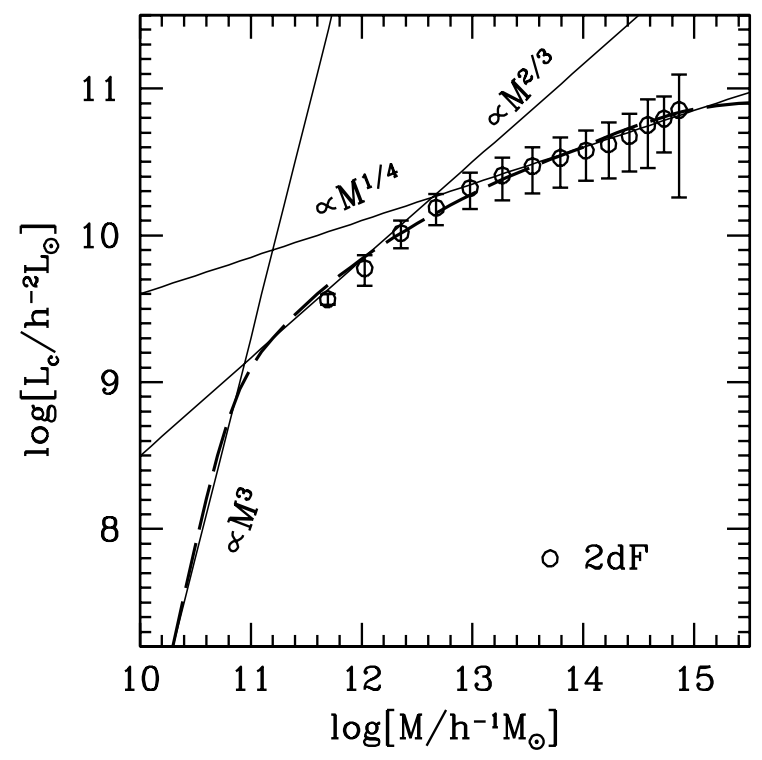

Figure 2. The mean central galaxy luminosity, $L_{c}$, as function of halo mass, $M$, over a large range in haloes masses. The data points are results obtained from galaxy groups (Yang et al. 2005b). The dashed curve is the $L_{c}-M$ relation given by the CLF obtained from matching the observed luminosity function of galaxies and the correlation length as a function of galaxy luminosity. This plot indicates that scaling relations such as the Tully-Fisher relation hold only over a limited range of halo masses.

characteristic scale in galaxy formation. This extremely tight confidence levels obtained from our CLF analysis suggests that we have established a robust connection between light and mass. Finally, the lower right-hand panel of Fig. 1 plots the corresponding mass-to-light ratios as function of halo mass. The pronounced minimum in $\langle M / L\rangle_{M}$ indicates that galaxy formation is the most efficient in haloes with masses in the range $5 \times$ $10^{10} h^{-1} \mathrm{M}_{\odot}<M<10^{12} h^{-1} \mathrm{M}_{\odot}$. For less massive haloes, $\langle M / L\rangle_{M}$ increases drastically with decreasing halo mass, which is required in order to bring the steep slope of the halo mass function at low $M$ in agreement with the relatively shallow faint-end slope of the observed LF. It indicates that galaxy formation needs to become extremely inefficient in haloes with $M<5 \times 10^{10} h^{-1} \mathrm{M}_{\odot}$ in order to prevent an overabundance of faint galaxies. The increase in $\langle M / L\rangle_{M}$ from $M \sim 10^{11} h^{-1} \mathrm{M}_{\odot}$ to $M \sim 10^{14} h^{-1} \mathrm{M}_{\odot}$ is thought to be associated with the decreasing ability of the gas to cool with increasing halo mass. The open circles with errorbars correspond to the semi-analytical model of Benson et al.(2002), which has been tuned to match the galaxy luminosity function. It is extremely reassuring that two completely independent methods yield average mass-tolight ratios that are in such good agreement.

Because it gives a statistical description of the galaxy-dark matter connection, the CLF is an extremely powerful tool. In a series of papers we have used it to to construct realistic mock samples (Yang et al. 2004; Weinmann et al. 2006), and to investigate large scale structure (Yang et al. 2004), the environment dependence of the galaxy luminosity function (Mo et al. 2004), the kinematics and abundances of satellite galaxies (van den Bosch et al. 2004, 2005, and various properties of galaxy groups (Yang et al. 2005b). In addition, we have used the CLF formalism to constrain cosmological parameters (van den Bosch et al. 2003b). 


\section{Galaxy Groups}

To use galaxy systems, such as galaxy groups and clusters (referred together as galaxy groups) to represent dark matter halos, one needs a group finder that can successfully group observed galaxies together according to common dark halos. We have developed a halo-based group finder that has such property (Yang et al. 2005a). The group finder starts with an assumed mass-to-light ratio to assign a tentative mass to each potential group. This mass is used to estimate the size and velocity dispersion of the underlying halo that hosts the group, which in turn is used to determine group membership (in redshift space). This procedure is iterated until group memberships converge. Detailed tests with our MGRS show that this group finder (i) is $>90 \%$ complete in terms of group membership, (ii) yields interloper fractions $<20 \%$, and (iii) yields group catalogues that are insensitive to the initial assumption of the mass-to-light ratios. Group masses, $M$, are determined by computing the mean separation between all groups brighter than the group under consideration and matching this with the mean separation between dark matter haloes more massive than $M$.

We have applied this group finder to both the the 2dFGRS (Yang et al. 2005a) and the SDSS (Weinmann et al. 2006). Fig. 2 plot the relation between the luminosity of the brightest (central) galaxy in each group, $L_{c}$, and the group (halo) mass, $M$ for the $2 \mathrm{dFGRS}$ sample. The mean $L_{c}-M$ relation is remarkably similar to that obtained from the CLF approach (dashed curve), and well described by a broken power-law with $L_{c} \propto M^{2 / 3}$ at $M<10^{13} h^{-1} M_{\odot}$ and $L_{c} \propto M^{1 / 4}$ at $M>10^{13} h^{-1} M_{\odot}$. At the low-mass end, this is in excellent agreement with results based on galaxy-galaxy weak lensing (e.g., Yang et al. $2003 \mathrm{~b})$. At the massive end, $L_{c}$ only increases very slowly with halo mass, indicating that there must be a physical process that prevents the central galaxies in massive haloes from growing. Finally, we want to emphasize that the groups so constructed can also be used to directly measure the halo (= group) occupation statistics, such as the CLF (Yang et al. 2005b), the properties of galaxies in halos of different masses (Weinmann et al. 2006), the spatial distribution of satellite galaxies in dark halos (Yang et al. 2005c), and the alignment between central galaxy and the distribution of satellite galaxies (Yang et al. 2006).

\section{References}

Benson A.J., Lacey C.G., Baugh C.M., Cole S., \& Frenk C.S., 2002, MNRAS, 333, 156

Madgwick D.S., et al., 2002, MNRAS, 333, 133

Mo H.J., \& White S.D.M., 1996, MNRAS, 282, 347

Mo H.J., Yang X., van den Bosch, F.C., \& Jing Y.P., 2004, MNRAS, 349, 205

Norberg P., et al., 2002, MNRAS, 332, 827

Vale A., \& Ostriker J.P., 2004, MNRAS, 353, 189

van den Bosch F.C., Yang X.H., \& Mo H.J., 2003a, MNRAS, 340, 771

van den Bosch F.C., Mo H.J., \& Yang X.H., 2003b, MNRAS, 345, 923

van den Bosch F.C., Norberg P., Mo H.J., \& Yang X.H., 2004, MNRAS, 352, 1302

van den Bosch F.C., Yang X.H., Mo H.J., \& Norberg P., 2005, MNRAS, 356, 1233

Weinmann S.M., van den Bosch F.C., Yang X., \& Mo H.J., 2006, MNRAS, 366, 2

Yang X.H., Mo H.J., \& van den Bosch F.C., 2003a, MNRAS , 339, 1057

Yang X.H., Mo H.J., Kauffmann Guinevere, \& Chu Y.Q., 2003b, MNRAS, 339, 387

Yang X.H., Mo H.J., Jing Y.P., \& van den Bosch F.C., Chu Y.Q., 2004, MNRAS , 350, 1153

Yang X.H., Mo H.J., van den Bosch F.C., \& Jing Y.P., 2005a, MNRAS, 356, 1293

Yang X.H., Mo H.J., Jing Y.P., \& van den Bosch F.C., 2005b, MNRAS, 358, 217

Yang X.H., Mo H.J., van den Bosch F.C., \& Jing Y.P., 2005c, MNRAS, 362, 711

Yang X., et al., 2006, MNRAS, 369, 1293 


\section{Discussion}

MARC VerheiJen: A comment: you can save yourself a lot of trouble by considering the K-band Tully-Fisher relation instead of the B-band, which suffers from stellar population issues and uncertainties in correction for internal extinction. It is also important to realize the kinematic meaning of the observed linewidths, as it does not necessarily reflect the depth of the dark matter halo potential. In general I would like to urge the simulators to be more critical to all the different observed TF-relations that exist in the literature. Ask yourself the question whether your "cobserved" $d$ TF-relation of choice is actually compatible with your simulated TF-relation.

\section{Houjun Mo: OK}

MARC VERHEIJEN: The CLF implies that a galaxy with a certain luminosity may reside in a DM halo with a range of masses. Is this consistent with the low scatter in the TF relation?

Houjun Mo: If you look at central galaxies, the scatter is consistent with TF-relation around $\mathrm{L}^{*}$. The scatter becomes bigger for faint galaxies. It is unclear if there is a problem here, since the TF scatter for faint galaxies is uncertain.

Ben Moore: How many $10^{10} \mathrm{~L}_{\odot}$ galaxies do you know that have $\mathrm{M} / \mathrm{L}=10000$ ?

Houjun Mo: For $10^{10} \mathrm{~L}_{\odot}$ galaxies, we obtain $\mathrm{M} / \mathrm{L}=100$. If you mean halos with $10^{10}$ $\mathrm{M}_{\odot}$, the conditional luminosity function model does predict a mean $\mathrm{M} / \mathrm{L}=10000$. If CDM model is correct, we do need to have many low-mass haloes undetected. The detected ones may be those that somehow managed to form enough stars. 